\title{
Formulation of lacto-fermented orange juice with incorporation of dairy whey
}

Shruti Yadav, Kursheed Alam Khan and Vijay Agrawal

Received : $10.04 .2018 ;$ Accepted : 13.04 .2018

See end of the Paper for authors' affiliation

Correspondence to :

\section{Shruti Yadav}

Agricultural Engineering Division, Indian Council Agricultural Research, Pusa Campus, New Delhi (India)
- Abstract : Disposal of whey is a major problem in the dairy industry, but the whey can be one of the source for the lactic acid production. Production of lactic acid through the Lactobacillus sp. could be a processing route for whey lactose and various attempts have been made in this direction. The main objective of the study is the formulation of lacto-fermented orange juice using whey. The method which followed is the fermentation process using whey as a starter culture. Fully mature oranges were procured from the market and juice was extracted with juicer available in the laboratory. The blends of whey (used as a starter culture), fresh orange juice, salt and purified water were prepared. Theses blends were put in the tightly covered jars at ambient condition for 48 hours and then refrigerated for 15 days, after this transferred to the other jar for storage studies at regular interval of three months. The microbiological (TPC, Coliform, Yeast and mold and Salmonella), chemical (TSS, Acidity, Ascorbic Acid, Sodium and Potassium), heavy metals (Arsenic, Lead, Tin, Zinc and Copper) testing and organoleptic analysis were performed for judging the shelf-life of the blends. Small colonies of approximately $1 \mathrm{~mm}$ diameter, lenticular, white or milky in color were observed on a solid medium. No pink color colonies confirmed the absence of coliform bacteria and also negative for the salmonella and yeast and mold colonies. The further microscopic examination reveals that the strains were gram positive with a cellular rod in pairs and chains which were further identified for the presence of lactic acid bacteria. The TSS, acidity, and ascorbic acid content of the blend was $7 \%, 2.5 \%$ and $250 \mathrm{ppm}$, respectively. The heavy metals such as tin, zinc, and copper in the blend were $50 \mathrm{ppm}, 3 \mathrm{ppm}$ and $2 \mathrm{ppm}$, respectively whereas heavy metals such as arsenic and lead were not found in the sample. During storage studies, each sample was also tested for color, texture, and taste. It shows that the fermented juice can be consumed within 15 days, whereas the juice kept in closed container can be consumed within 9 months.

- Key words : Lacto-fermented orange juice, Dairy whey, Lactic acid

- How to cite this paper : Yadav, Shruti, Khan, Kursheed Alam and Agrawal, Vijay (2018). Formulation of lacto-fermented orange juice with incorporation of dairy whey. Internat. J. Agric. Engg., 11(Sp. Issue) : 29-36, DOI: 10.15740/HAS/IJAE/11.Sp. Issue/29-36. 